\title{
artigo
}

Evangelista, W.A.; Vasconcelos, E.E.C.; Brito, D.G.C.; Alburquerque, L.S.S.; Rodrigues, L.G.L.; Souza Silva, L.G.

Cuidados de enfermagem na cirurgia cardíaca: perspectivas da literatura atual

\section{Cuidados de enfermagem na cirurgia cardíaca: perspectivas da literatura atual}

\author{
Nursing care in heart surgery: perspectives of current literature
}

Atención de enfermería en cirugía cardíaca: perspectivas de la literatura actual

\begin{abstract}
RESUMO
Objetivo: analisar as publicações científicas acerca dos cuidados de enfermagem ao paciente de cirurgia cardíaca. Método: Trata-se de uma revisão integrativa da literatura, da modalidade exploratória, descritiva, com abordagem qualitativa. Foi realizada no período de agosto a outubro de 2020, nas seguintes bases de dados, indexadas a Biblioteca Virtual de Saúde: SciELO, LILACS e MEDLINE. Foram selecionadas 07 publicações, e estas foram analisadas, e organizadas em dois quadros, contendo: título, autores, ano de publicação, e os cuidados de enfermagem na cirurgia cardíaca. Resultados: 0 enfermeiro, junto com sua equipe, acompanha o paciente em todas as etapas da cirurgia cardíaca, e realiza cuidados na alimentação, orientação e acolhimento da família, esclarecimentos para o procedimento, exame físico e sistematização da assistência de enfermagem. Conclusão: os cuidados de enfermagem são essenciais na cirurgia cardíaca, e que ainda é necessário novas pesquisas que fortaleçam e evidenciem esse cuidado.
\end{abstract}

DESCRITORES: Enfermagem; Cirurgia cardíaca; Cuidado; Enfermagem.

\section{ABSTRACT}

Objective: to analyze scientific publications about nursing care for cardiac surgery patients. Method: This is an integrative literature review, of the exploratory, descriptive modality, with a qualitative approach. It was carried out from August to October 2020, in the following databases, indexed to the Virtual Health Library: SciELO, LILACS and MEDLINE. Seven publications were selected, and these were analyzed, and organized in two tables, containing: title, authors, year of publication, periodical and nursing care in cardiac surgery. Results: The nurse, along with his team, accompanies the patient in all stages of cardiac surgery, and performs care in feeding, guidance and welcoming the family, clarifications for the procedure, physical examination and systematization of nursing care. Conclusion: nursing care is essential in cardiac surgery, and further research is needed to strengthen and highlight this care.

DESCRIPTORS: Nursing; Cardiac surgery; Watch out; Nursing.

\section{RESUMEN}

Objetivo: analizar publicaciones científicas sobre cuidados de enfermería al paciente de cirugía cardíaca. Método: Se trata de una revisión bibliográfica integradora, de modalidad exploratoria, descriptiva, con enfoque cualitativo. Se realizó de agosto a octubre de 2020, en las siguientes bases de datos, indexadas a la Biblioteca Virtual en Salud: SciELO, LILACS y MEDLINE. Se seleccionaron siete publicaciones, que fueron analizadas y organizadas en dois tablas, conteniendo: título, autores, año de publicación, asistencia periódica y de enfermería en cirugía cardíaca. Resultados: La enfermera, junto con su equipo, acompaña al paciente en todas las etapas de la cirugía cardíaca, y realiza cuidados en la alimentación, orientación y acogida de la familia, aclaraciones para el procedimiento, exploración física y sistematización de los cuidados de enfermería. Conclusión: el cuidado de enfermería es fundamental en la cirugía cardíaca, y se necesita más investigación para fortalecer y resaltar este cuidado.

DESCRIPTORES: Enfermería; Cirugía cardíaca; Cuidado; Enfermería.

RECEBIDO EM: 08/12/2020 APROVADO EM: 04/01/2021

\section{Wanessa de Araújo Evangelista}

Enfermeira (UNINASSAU João Pessoa), Pós-graduanda em Emergência e UTI (FESVIP), Pós-graduanda em Instrumentação Cirúrgica, Centro Cirúrgico e Central de Material e Esterilização (FAVENI).

ORCID: 0000-0002-5702-5634 


\section{Eduarda Ellen Costa Vasconcelos}

Enfermeira. Centro Universitário de João Pessoa Unipê. Pós-graduanda em Gerontologia pelo grupo Excelência Cursos. ORCID: 0000-0001-8971-2917

\section{Dayse Gadioli Cavalcante de Brito}

Enfermeira. Centro Universitário de João Pessoa Unipê. ORCID: 0000-0003-0510-8566

\section{Luciene de Souza Santos Albuquerque}

Enfermeira. Centro Universitário de João Pessoa Unipê. ORCID: 0000-0001-5119-195X

\section{Luana Gomes Leitão Rodrigues}

Enfermeira. Centro Universitário de João Pessoa Unipê. ORCID: 0000-0001-5259-1357

\section{Lúcia Gomes de Souza Silva}

Bacharelado em Enfermagem. Pós-graduação em Terapia Intensiva e em Urgência e Emergência.

ORCID: 0000-0001-6998-435X

\section{INTRODUÇÃO}

D entre as doenças crônicas não transmissíveis, encontram-se as doenças cardiovasculares, que são umas das principais causas de morte no Brasil e no mundo, gerando uma grave situação nos sistemas de saúde. A grande maioria destas doenças estão associadas a fatores comportamentais de risco, como o tabagismo, sedentarismo, uso abusivo de álcool, alimentação inadequada e obesidade, sendo um alerta para medidas de prevenção e tratamento deste grande agravo de saúde mundial ${ }^{(1)}$.

Em alguns casos de pessoas com doenças cardiovasculares, a mudança no estilo de vida e o uso de medicações não são suficientes para que o coração desempenhe seu papel corretamente, sendo necessário intervenção cirúrgica. A cirurgia cardíaca tem como objetivo restabelecer as funções do coração, melhorando a qualidade de vida dos pacientes, diminuindo os sintomas e aumentando a sobrevida ${ }^{(2)}$.

A cirurgia cardíaca trata-se de um procedimento complexo realizado por uma equipe multiprofissional. Esta intervenção acarretaem alterações de vários mecanismos fisiológicos impondo constantes mudanças de ordem física, social e psicológica, compreendendo um momento difícil para o paciente cardiopata, sendo de fundamental importância que o enfermeiro e a equipe multiprofissional estejam preparados para apoiar, orientar e avaliar suas necessidades, permitindo uma vivência diante do processo saúde-doença menos desgastante ${ }^{(3)}$.

Com o avanço da cirurgia cardíaca gerou-se a necessidade de expansão e desenvolvimento dos cuidados de enfermagem prestados aos pacientes submetidos a este tipo de cirurgia. Os cuidados de enfermagem são fundamentais na cirurgia cardíaca, visando um atendimento integral e individualizado, atendendo todas as necessidades físicas e psíquicas do paciente, assegurando a qualidade do processo operatório ${ }^{(4)}$.

A Sistematização da Assistência de Enfermagem Perioperatória (SAEP) direciona a atuação do enfermeiro no desempenho de suas atividades profissionais, simplificando o desenvolvimento da assistência de forma segura, integral, individualizada e contínua, estabelecendo um serviço de qualidade, além de prevenir e reduzir a incidência de acontecimentos inadequados referentes a assistência prestada. É um processo constituído pelas seguintes etapas: visita pré-operatória, planejamento da assistência perioperatória, implementação da assistência, avaliação da assistência (visita pós-operatória de enfermagem) e reformulação da assistência a ser prestada. Estas etapas orientam a atuação do enfermeiro nos períodos pré-operatório, transoperatório e pós-operatório ${ }^{(5)}$.

Com base nos dados expostos, levantou-se a seguinte questão de pesquisa: quais as evidências científicas disponíveis na literatura atual sobre os cuidados de enfermagem ao paciente submetido a cirurgia cardíaca? Deste modo, afim de contribuir e somar esforços para melhoria dos cuidados de enfermagem, o presente estudo tem como objetivo analisar as publicações científicas acerca dos cuidados de enfermagem ao paciente de cirurgia cardíaca.

\section{MÉTODO}

O presente estudo trata-se de uma revisão integrativa da literatura, que viabiliza a síntese e a análise do conhecimento científico já elaborado sobre a temática investigada. Para conduzir a pesquisa foram percorridas as seguintes etapas: identificação do problema e elaboração da questão de pesquisa; definição dos critérios de inclusão e exclusão para busca na literatura; avaliação de dados; interpretação dos resultados e conclusões; apresentação da revisão integrativa ${ }^{(6)}$.

Para elaboração da questão de pesquisa da revisão integrativa, foi utilizada a estratégia metodológica PICO (acrônimo para patient, intervention, comparison, outco- 
mes). Nesta pesquisa o P-consiste no paciente de cirurgia cardíaca, I- refere-se aos cuidados de enfermagem para esse grupo, C- analisa, compara e caracteriza as publicações científicas sobre os cuidados de enfermagem na cirurgia cardíaca e O- espera que os pacientes submetidos à cirurgia cardíaca tenham recebido os cuidados de enfermagem de forma efetiva, e que esse cuidado seja essencial.

A busca na literatura por publicações referentes aos cuidados de enfermagem na cirurgia cardíaca, foi realizada no período de agosto a outubro de 2020, nas seguintes bases de dados, frequentemente utilizadas na área da saúde e indexadas à Biblioteca Virtual de Saúde: SciELO- Scientific Electronic Library Online, LILACS- Latino-Americana de informação bibliográfica em ciências da saúde e MEDLINE-Sistema Online de Busca e Análise de Literatura Médica.

Os descritores utilizados para coleta de dados foram extraídos do Descritores em Ciências da Saúde (DECs), que são: Enfermagem; Cirurgia cardíaca; Enfermagem e Cuidado, cruzados pelo operador booleano AND. Destaca-se que o termo "cirurgia cardíaca" é descrito nos Descritores em Ciências da Saúde (DECs) como sinônimo de "cirurgia torácica", mas que permite usar o termo "Cirurgia cardíaca" ou ainda "Cirurgia do coração" como alternativas para busca.

Os critérios de inclusão definidos, constituíram-se de artigos publicados no período de 2015 a 2020, em língua portuguesa, disponíveis integralmente, de forma gratuita, artigos originais que responderam à questão de pesquisa e que estavam condizentes com o objetivo da pesquisa. E como critérios de exclusão: artigos fora da linha temporal estabelecida; artigos em outro idioma; artigos de outras bases de dados; artigos fora do contexto da temática (objetivo e questão de pesquisa) e artigos em duplicidade no momento da busca nas bases de dados.

Dessa forma, foram encontradas 606 publicações que envolviam os cuidados de enfermagem na cirurgia cardíaca, destas, 147 publicações possuíam títulos correspondentes a temática e 138 publicações encontravam-se no período de 2015 a 2020 , mas apenas 43 publicações respondiam ao objetivo e questão de pesquisa. Estas 43 publicações tiveram os resumos lidos e analisados, e somente 20 publicações foram selecionadas para a leitura integral, as demais foram excluídas, por não tratarem da temática de forma direta, abrangendo o objetivo do estudo. Estas 20 publicações foram lidas integralmente, e 07 publicações estavam nos critérios de inclusão estabelecidos, responderam aos objetivos, a questão de pesquisa e a temática abordada. No total, foram excluídas 599 publicações, fazendo parte do corpo de análise dos resultados desta pesquisa 07 publicações científicas. As publicações foram analisadas e organizadas em quadros para melhor apreciação.

\section{RESULTADOS E DISCUSSÃO}

As publicações foram organizadas em dois quadros, que abordaram as seguintes variáveis: título da publicação, autor, ano, e os cuidados de enfermagem na cirurgia cardíaca encontrados nas publicações. As publicações foram codificadas a partir da letra "P", seguindo a numeração de 1 a 7 , P1; P2...e assim sucessivamente.

Diante do quadro 1, estão organizados os títulos, onde é possível perceber, de forma inicial, alguns cuidados já descritos, que apontam desde o uso de teorias de enfermagem para auxiliar no cuidado, até o uso da religião e espiritualidade como fonte de enfrentamento para o paciente e recurso nos cuidados de enfermagem.

A respeito do ano de publicação, os anos de 2018, 2017 e 2015, obtiveram duas publicações, estando em ascendência sobre os

\begin{tabular}{|c|c|c|c|}
\hline CODIFICAÇÃO & TÍTULO DA PUBLICAÇÃO & AUTORES & ANO \\
\hline P1 & $\begin{array}{l}\text { Contribuição do cuidado transpessoal ao ser-cardiopata no pós-opera- } \\
\text { tório de cirurgia cardíaca. }\end{array}$ & $\begin{array}{l}\text { RABELO, A.C.S; SOUZA, F.V.F.S, } \\
\text { SILVA, L.F }{ }^{(7)} \text {. }\end{array}$ & 2017. \\
\hline P2 & $\begin{array}{l}\text { Temporalidade da mulher após cirurgia cardíaca: contribuições para } \\
\text { o cuidado de enfermagem. }\end{array}$ & AMORIM, T.V.et al(8). & 2015. \\
\hline P3 & $\begin{array}{l}\text { Considerações de pacientes no perioperatório de cirurgia cardíaca refe- } \\
\text { rentes às orientações recebidos do enfermeiro. }\end{array}$ & $\begin{array}{l}\text { COPPETTI, L.C.de; STUMM, E.M.F; } \\
\text { BENETTI, E.R.R'(9). }\end{array}$ & 2015. \\
\hline P4 & $\begin{array}{l}\text { Caminho percorrido até a cirurgia cardíaca: necessidades e expectativas } \\
\text { no pré-operatório. }\end{array}$ & KNIHS, N.S.da. et al(10). & 2017. \\
\hline P5 & $\begin{array}{l}\text { Protocolo de enfermagem no implante de valva aórtica transcateter: } \\
\text { um direcionamento para o cuidado. }\end{array}$ & SILVA, G.S.da ${ }^{(11)}$. & 2018. \\
\hline P6 & $\begin{array}{l}\text { Percepção dos cuidadores frente à humanização da assistência no } \\
\text { pós-operatório imediato de cirurgia cardíaca. }\end{array}$ & $\begin{array}{l}\text { MILANI, P; LANFERDINI, I.Z; ALVES, } \\
\text { V.B. et al al(12). }\end{array}$ & 2018. \\
\hline P7 & $\begin{array}{l}\text { Religiosidade, bem-estar espiritual e cuidado transpessoal no pré-ope- } \\
\text { ratório de cirurgia cardíaca. }\end{array}$ & GOMES, E.T; BEZERRA, S.M.M.S.da ${ }^{(13)}$. & 2020. \\
\hline
\end{tabular}


demais períodos. $\mathrm{O}$ ano de 2020 teve uma publicação, e os anos de 2016 e 2019 não obtiveram publicações referentes a temática dentro dos critérios de inclusão.

É perceptível que o enfermeiro precisa ultrapassar o seu olhar técnico, e às vezes mecânico para o cuidado, e olhar o paciente de forma integral, considerando o seu bem-estar completo, inclusive espiritual $^{(7)}$. O enfermeiro junto com sua equipe acompanha o paciente e todas as etapas da cirurgia, e realiza cuidados na alimentação, orientação e acolhimento da família, esclarecimentos para o paciente sobre o seu procedimento, exame físico, sistema- tização da assistência de enfermagem, e outros procedimentos que são primordiais para consolidar a cirurgia cardíaca ${ }^{(10)}$.

Dessa forma, o cuidado de enfermagem, em todo o processo cirúrgico cardíaco, precisa atender as necessidades do paciente, conforme a sua complexidade humana $^{(7)}$. E o enfermeiro, junto a sua equipe, é fundamental nesse momento, onde deve estar apto e ativo para auxiliar o paciente em suas demandas ${ }^{(8)}$.

Como explanado nos artigos analisados, é de responsabilidade da equipe de saúde e enfermagem, orientar o paciente sobre o procedimento, cuidando do mesmo de forma completa, desde as orientações alimentares, até aquelas que correspondem a higiene pessoal ${ }^{(9)}$. É preciso entender, que mediante as orientações recebidas, os pacientes de cirurgia cardíaca sentem-se seguros, e compreendem o momento que irão passar, além de reduzir a ansiedade, e conseguir identificar sentimentos nas linguagens corporais, e muitas vezes não-verbais ${ }^{(9)}$.

Sabe-se que o procedimento cirúrgico em si, gera diversos sentimentos no paciente, e contribuem para a angústia, insegurança, medo, ansiedade e outros sentimentos ocasionados pelo momento. $\mathrm{O}$ enfermeiro precisa implementar ações de

\section{QUADRO 2: Apresentação e caracterização dos cuidados de enfermagem na cirurgia cardíaca encontrados nas} publicações científicas:

\begin{tabular}{|c|c|}
\hline CODIFICAÇÃO & CUIDADOS DE ENFERMAGEM NA CIRURGIA CARDÍACA \\
\hline P1 & $\begin{array}{l}\text { *O enfermeiro deve ser capaz de transcender o contexto físico, buscando o espiritual do paciente, cuidado de forma } \\
\text { biopsicossocial. } \\
\text { *É considerado cuidado de enfermagem individualizado, quando é contínuo, planejado, com diversas fases, direcionado } \\
\text { para apenas um paciente, sabendo que em situações como a cirurgia cardíaca, o cuidado é singular, sendo relevante a } \\
\text { avaliação pré-operatória, transoperatória e pós-operatória. } \\
\text { *É preciso realizar o acolhimento do paciente com amor, proporcionar um ambiente tranquilo, e uma boa recuperação ao } \\
\text { paciente de cirurgia cardíaca. } \\
\text { *A equipe de enfermagem precisa oferecer confiança, formar vínculo, criar um elo com a família e o paciente, levando em } \\
\text { consideração a complexidade cirúrgica. }\end{array}$ \\
\hline P2 & $\begin{array}{l}\text { *A importância dos cuidados de forma integral oferecidos pela enfermagem. } \\
\text { *Para alcançar essa integralidade nos cuidados, os enfermeiros precisam se comunicar melhor. } \\
\text { *O enfermeiro também pode usar da educação em saúde, e inserir as famílias dos pacientes nos cuidados a serem } \\
\text { realizados. }\end{array}$ \\
\hline P3 & $\begin{array}{l}\text { *Dentre os cuidados de enfermagem, percebeu-se que as orientações quanto ao procedimento cirúrgico em si, aneste- } \\
\text { sia, cuidados físicos e pós-operatório tiveram destaque. } \\
\text { *Outro cuidado importante, é que cabe ao enfermeiro identificar e compreender a comunicação não verbal do paciente, } \\
\text { que pode sinalizar ansiedade, angústia com o procedimento e outros sentimentos importante. }\end{array}$ \\
\hline P4 & $\begin{array}{l}\text { *O enfermeiro direciona as ações para o cuidado, com o objetivo de atender as necessidades do paciente cardíaco que } \\
\text { está no período pré-operatório, e isso implica diretamente na redução do risco cirúrgico. } \\
\text { *Um plano de intervenções singular para o processo operatório do paciente cardíaco é primordial, que consiga suprir as } \\
\text { necessidades de cuidado. } \\
{ }^{*} \text { Os cuidados de enfermagem devem ser planejados, sistematizados e organizados. }\end{array}$ \\
\hline P5 & $\begin{array}{l}\text { *Quando a enfermagem segue um protocolo, possibilita a segurança do paciente na cirurgia cardíaca e em demais pro- } \\
\text { cedimentos a serem realizados. } \\
\text { *Os enfermeiros contribuem realizando exame físico, identificado alterações respiratórias, acompanhando o tratamento, } \\
\text { e outros cuidados importantes. }\end{array}$ \\
\hline P6 & $\begin{array}{l}\text { *O pós-operatório de cirurgia cardíaca exige da equipe de enfermagem observação contínua, por ser uma atividade que } \\
\text { exige atenção e cuidado de alta complexidade. } \\
{ }^{*} \text { equipe de enfermagem possibilita a redução de angústias e inquietações. }\end{array}$ \\
\hline P7 & $\begin{array}{l}\text { *Com base na teoria do cuidado transpessoal, o artigo aponta que a espiritualidade é importante para a compreensão de } \\
\text { momentos críticos, assim como na cirurgia cardíaca. } \\
{ }^{*} \text { A espiritualidade é uma estratégia de enfrentamento durante a cirurgia cardíaca, e auxilia em momentos difíceis. }\end{array}$ \\
\hline
\end{tabular}


cuidado que mostrem que aquele procedimento é realmente necessário, trazendo segurança para o paciente e sua famíliaa ${ }^{(10)}$.

Sistematizar a assistência, planejar o cuidado, organizar as ações de enfermagem, realizar procedimentos terapêuticos, são funções da enfermagem que implicam diretamente na qualidade de vida do paciente em cirurgia cardíaca. Não se pode esquecer, que os cuidados de enfermagem são singulares, direcionados para cada indivíduo de forma diferente, com objetivos e resultados distintos, sempre incluindo a família e com condutas embasadas cientificamente ${ }^{(11)}$.

A espiritualidade é um recurso, fonte de fé para o cuidado ao paciente em cirurgia cardíaca, e é considerada relevante para auxi- liar o paciente no procedimento, resultando em menores agravos no pós-operatório ${ }^{(13)}$. Além da espiritualidade, é preciso acolher os familiares, fornecer apoio, confiança, formar vínculo, e sempre prestar as informações necessárias, pois, é ideal que o profissional esteja próximo aos familiares, esclarecendo as dúvidas acerca do procedimento ${ }^{(12)}$.

\section{CONSIDERAÇÕES FINAIS}

Esta pesquisa buscou, evidenciou e alcançou os seus objetivos propostos inicialmente. Percebeu-se a importância dos cuidados de enfermagem na cirurgia cardíaca, e o quanto estes são essenciais para a efetividade da cirurgia.
Dentre os cuidados elencados, a redução da ansiedade, o planejamento, sistematização das ações, estabelecimento da confiança entre profissional/ paciente/família, acolhimento e boa comunicação da equipe com o paciente, foram resultados descritos nas publicações analisadas.

Desse modo, faz-se necessário novas pesquisas dentro da temática, que tragam outros resultados, caminhos e perspectivas para qualificar o cuidado de enfermagem ao paciente cardíaco. Recomenda-se a inserção de atualidades na literatura, para ampliar o conhecimento dos profissionais de enfermagem e da saúde, melhorando a prática laboral dos mesmos.

\section{REFERÊNCIAS}

1. Sociedade Brasileira de Cardiologia-SBC. Cardiômetro. [internet] 2018 [cited 2020 out 25]. Available from: http://www.cardiometro.com.br/default.asp.

2. Organização Pan-Americana da Saúde/OPAS/OMS. Doenças Cardiovasculares. [internet] 2017 [cited 2020 out 25] Available from: https://www.paho.org/bra/index.php?option=com_ content\&view=article\&id=5253: doencas-cardiovasculares\&Itemid=1096.

3. Brasil. Ministério da Saúde - MS. Cardiologia/Cirurgia Cardíaca. [internet] 2017. [cited 2020 mai 25] Available from: https:// www.saude.gov.br/atencao-especializada-e-hospitalar/especialidades/cardiologia-cirurgia-cardiaca.

4. Pereira DA, Ferreira TM, Silva Jl, Gomes ET, Bezerra SMMS. Necessidades de aprendizagem acerca da cirurgia cardíaca na perspectiva de pacientes e enfermeiros. Rev. SOBECC. [Internet] 2018 [cited 2020 mai 30] 23 (2):84-88. Available from: https:// revista.sobecc.org.br/sobecc/article/view/399/pdf_1

5. Jost MT, Branco A, Viegas K, Caregnato RCA. Sistematização da assistência de enfermagem perioperatória: avaliando os processos de trabalho no transoperatório. Enferm. foco. [internet] 2019 [cited 2020 mai 30] 10 (2):43-49. Available from: https:// pesquisa.bvsalud.org/portal/resource/pt/biblio-1050811.

6. Brasil. Ministério da Saúde. Diretrizes Metodológicas: elaboração de revisão sistemática e metanálise de estudos randomizados. [internet] 2012 [cited 2020 out 25]. Available from:http:// bvsms.saude.gov.br/bvs/publicacoes/diretrizes_metodologicas_elaboracao_sistematica.pdf.

7. Rabelo ACS, Souza FVFS, Silva LF. Contribuição do cuidado transpessoal ao ser-cardiopata no pós-operatório de cirurgia cardíaca. Rev Gaúcha Enferm. [internet] 2017 [cited 2020 out 20] 38(4):e64743. Available from: https://www.scielo.br/pdf/ rgenf/v38n4/1983-1447-rgenf-38-04-e64743.pdf.
8. Amorim TV, Salimena AMO, Souza IEO, Melo MCSC, Silva LF, Cadete MMM. Temporalidade da mulher após cirurgia cardíaca: contribuições para o cuidado de enfermagem. Rev. Bras. Enferm. [Internet] 2015 [cited 2020 out 25] 68 (6):1056-1062, Dec. 2015. Available from:http://www.scielo.br/scielo.php?script=sci_arttext\&pid=S0034-71672015000601056\&lng=en\&nrm=isso.

9. Coppetti LC de, Stumm EMF, Benetti ERR. Considerações de pacientes no perioperatório de cirurgia cardíaca referentes às orientações recebidas do enfermeiro. Rev Min Enferm. [internet] 2015 [cited 2020 out 23] 19(1): 113-119. Available from: https:// cdn.publisher.gn1.link/reme.org.br/pdf/v19n1a10.pdf.

10. Knihs NS da, Valmorbida AP, Lanzoni GMM, Roza BA, Ghellere A. Caminho percorrido até a cirurgia cardíaca: necessidades e expectativas no pré-operatório. Av.enferm. [internet] 2017 [cited 2020 out 22] 35(1):30-41. Available from:http://www.scielo.org.co/scielo.php?script=sci_arttext\&pid=S0121-45002017000100004\&Ing=en\&nrm=iso.

11. Silva GC da, Albuquerque DC, Rocha RG, Fernandes RTP, Lima LCLC, Cabral APV. Nursing protocol in transcatheter aortic valve implantation: care guideline. Esc. Anna Nery. [internet] 2018 [cited 2020 out 21] 22 (3) e20170260. Available from: http://www.revenf.bvs.br/scielo.php?script=sci_arttext\&pid=S141481452018000300212\&Ing=pt\&nrm=iso.

12. Milani P, Lanferdini IZ, Alves VB. Percepção dos Cuidadores Frente à Humanização da Assistência no Pós-Operatório Imediato de Cirurgia Cardíaca. J. res.: fundam. care. [internet] 2018 [cited 2020 out 21] 10(3): 810-816. Available from: http://www.seer.unirio.br/index.php/ cuidadofundamental/article/viewFile/6208/pdf_1.

13.Gomes ED, Bezerra SMMS da Religiosidade, bem-estar espiritual e cuidado transpessoal no pré-operatório de cirurgia cardíaca. Revista Cuidarte. [internet] 2020 [cited 2020 out 20] 11(2): e1020. Available from:https://revistacuidarte.udes.edu. co/index.php/cuidarte/article/view/1020/1546. 